\title{
Estudo experimental de reconstrução da veia femoral com enxerto tubular do bipolímero de cana-de-açúcar.
}

\section{Experimental study of femoral vein reconstruction with sugarcane biopolymer tubular graft.}

Daniella Maria Souza Silva'; Esdras Marques lins, TCBC-PE ${ }^{1}$; Sílvio Romero Barros Marques, TCBC-PE ${ }^{1}$; Amanda Vasconcelos Albuquerque ${ }^{1}$; José Lamartine Andrade Aguiar, TCBC-PE'1

R E S U M O

\begin{abstract}
Objetivo: avaliar, através de dopplerfluxometria, de venografia, de histologia e de evolução clínica, o uso de enxertos tubulares de biopolímero de cana-de-açúcar (BP) na reconstrução de veias femorais em cães. Métodos: oito cães adultos foram submetidos à reconstrução de veia femoral, à esquerda com enxerto tubular de BP e à direita com veia autóloga. No período pós-operatório, os animais foram submetidos à avaliação clínica e dopplerfluxometria das veias femorais. Após 360 dias, os cães foram reoperados e submetidos à flebografia das veias femorais com contraste iodado. Os segmentos das veias femorais contendo os enxertos foram retirados e enviados para avaliação histopatológica. Resultados: os cães não apresentaram hemorragia, hematoma, infecção da ferida operatória ou edema dos membros operados. Um animal apresentou dilatação venosa superficial na região inguinal esquerda. A flebografia realizada 360 dias após a primeira cirurgia demonstrou que três $(37,5 \%)$ enxertos de BP e sete $(87,5 \%)$ do grupo controle (C) estavam pérvios. Na avaliação histopatológica foi encontrada uma reação inflamatória com neutrófilos e linfócitos na superfície externa de ambos os grupos. Na camada íntima de revestimento dos enxertos e na camada externa nos dois grupos, foi encontrada fibrose. Conclusão: com base nos resultados obtidos com o modelo experimental utilizado, conclui-se que a BP apresenta potencial para ser utilizado como enxerto tubular para revascularização venosa, porém novas pesquisas precisam ser realizadas para confirmar a sua eficácia na revascularização de veias de médio e grande calibre, o que poderia permitir o seu uso na prática clínica.
\end{abstract}

Descritores: Enxerto Vascular. Veia Femoral. Saccharum. Bioprótese. Procedimentos Cirúrgicos Vasculares. Cães.

\section{INTRODUÇÃO}

cirurgia de reconstrução venosa, especialmente
quando envolve o uso de substitutos vasculares, representa um grande desafio ao cirurgião vascular já que as veias podem facilmente perder sua forma cilíndrica. Atualmente as técnicas cirúrgicas de reconstrução venosas mais utilizadas, empregam substitutos vasculares na forma tubular ou como patchs ${ }^{1-3}$.

Os substitutos vasculares usados na reconstrução venosa podem ser autólogos, biológicos ou sintéticos. Os substitutos autólogos apresentam o melhor custo-benefício, mas tem seu uso limitado pela disponibilidade de veias autólogas em cada paciente. Os substitutos sintéticos, tais como os de Dacron e e-PTFE apresentam alto custo, são menos resistentes à infecção e são utilizados na reconstrução das veias de grande calibre (-10 $^{\text {. }}$
Uma classe especial de material produzido a partir de substâncias sintetizadas por diferentes tipos de micro-organismos tem sido recentemente estudada. Esses micro-organismos apresentam características de tecidos orgânicos, tais como flexibilidade e resistência à ruptura. Um exemplo deste tipo de material é um polissacarídeo extracelular, produzido através da síntese bacteriana a partir do melaço da cana-de-açúcar, desenvolvido no Laboratório de Biopolímeros da Estação Experimental de Cana-de-Açúcar da Universidade Federal Rural de Pernambuco. O Biopolímero de Canade-Açúcar (BP) apresenta flexibilidade, resistência à ruptura além de ser biocompatível e apresentar baixa citotoxicidade ${ }^{11-17}$.

Recentemente a membrana de BP foi utilizada com sucesso como patch em arterioplastias e venoplastias nos vasos femorais de cães, não sendo relatados casos de trombose, ruptura ou rejeição ${ }^{16,17}$. O desenvolvimento na produção e a inovação tecnológica

1 - Universidade Federal de Pernambuco, Departamento de Cirurgia, Recife, PE, Brasil. 
dos materiais de BP resultaram na criação de tubos, que podem ser aplicados, com baixo custo, na área da cirurgia vascular como enxertos venosos tubulares.

O objetivo deste estudo foi avaliar, através da dopplerfluxometria, venografia, histologia e evolução clínica, o uso de enxertos tubulares de BP na reconstrução de veias femorais em cães.

\section{MÉTODOS}

Oito cães adultos, seis machos, com média de peso de 16,8Kg foram utilizados neste estudo. Os animais foram mantidos no Biotério do Núcleo de Cirurgia Experimental do Centro de Ciências da Saúde da Universidade Federal de Pernambuco (NCE-CCSUFPE). Esta pesquisa foi aprovada pelo Comitê de Ética em Experimentação Animal do Centro de Ciências Biológicas da Universidade Federal de Pernambuco (CEEA-UFPE), sob o processo no 23076.000105/201222, de acordo com as normas vigentes no Brasil, especialmente a Lei no 11.749 , de 2008, regulamentada pelo Decreto no 6899, de 15 de julho de 2009, que trata da questão do uso de animais para fins científicos.

Todos os animais antes de serem submetidos a qualquer procedimento cirúrgico receberam: 1) Duramune Max $10^{\circledR}$ para a imunização contra o vírus da cinomose, do adenovírus tipo 2 do coronavírus e do parvovírus CPV2b, do vírus da parainfluenza e contra as variações de Leptospira ( $L$. canicola, $L$. icterohaemorrhagiae, L. grippotyphosa e L. pomona; 2) vacina antirrábica. Os cães receberam como vermífugo, Ancilex ${ }^{\circledR}$ por via subcutânea, na dose de $1 \mathrm{ml} / 5 \mathrm{~kg}$, para tratamento das verminoses ancilostomíase, espirocercose e toxocaríase. Permaneceram no biotério sob vigilância por período de 21 dias antes de serem submetidos ao procedimento experimental. Foram alimentados, no pré-operatório e durante todo o período do experimento, diariamente com água e $6 \mathrm{Kg}$ de ração específica para cães adultos. O modelo de estudo empregado foi o experimental randomizado controlado.
O experimento foi dividido em dois grupos: grupo controle, constituído por oito veias femorais direitas com enxerto de veia femoral autóloga, e o grupo experimental, formado por oito veias femorais esquerdas com o enxerto tubular de BP. Nesta pesquisa cada animal foi o seu próprio controle. Durante o período do estudo, os animais foram conduzidos por técnicos e veterinários habilitados seguindo as normas internacionais para pesquisa com animais.

Antes da cirurgia os animais permaneceram em jejum por 12 horas. A anestesia foi realizada com tiopental sódico $(12,5 \mathrm{mg} / \mathrm{Kg})$, cloridrato de cetamina $(2,5 \mathrm{mg} / \mathrm{Kg})$ e brometo de pancurônio $(0,2 \mathrm{mg} / \mathrm{Kg})$. Cada animal foi então colocado em decúbito dorsal e a intubação orotraqueal foi realizada. A seguir, a tricotomia e a antissepsia das regiões inguinais foi realizada, e as veias femorais foram acessadas através de uma incisão cutânea inguinal longitudinal com $5 \mathrm{~cm}$ de comprimento. Após a exposição das veias femorais, um segmento de $10 \mathrm{~mm}$ de comprimento foi ressecado da veia femoral esquerda que, então, foi reconstruída com o enxerto tubular de BP, através de sutura contínua, término-terminal, proximal e distal com fio de polipropileno cardiovascular 7.0. A seguir, o mesmo procedimento foi realizado na veia femoral direita, mas sua reconstrução foi feita com $10 \mathrm{~mm}$ de segmento venoso retirado da veia femoral esquerda. Os cães foram acompanhados por 360 dias após a cirurgia através de avaliação clínica e da dopplerfluxometria.

Na primeira semana, os animais receberam avaliação clínica diária e, a partir do oitavo dia, a avaliação foi semanal até o 30 으 dia de pós-operatório. A partir de então, os animais foram avaliados mensalmente até o final do período de avaliação. Após 360 dias de monitoramento, os cães foram submetidos à nova intervenção cirúrgica seguindo as mesmas condições de anestesia e acesso das veias femorais da primeira cirurgia. Após a exposição das veias femorais e punção das veias safenas internas, direita e esquerda, uma flebografia foi realizada. Após a flebografia, os animais foram sacrificados com uma dose tóxica do anestésico 
e, então, foi realizada a retirada dos segmentos das veias femorais, esquerda e direita, contendo os enxertos do BP e do grupo controle.

Para a avaliação histológica, os segmentos venosos removidos foram fixados em formalina neutra a 10\%. O material foi então corado por hematoxilina e eosina, e tricrômicro de Masson, para estudo microscópico. Os espécimes foram processados no Laboratório de Pesquisa de Histopatologia da Pós-Graduação em Anatomia Patológica da Universidade Federal de Pernambuco.

Para análise dos dados foram obtidas distribuições absolutas e percentuais das medidas estatísticas, média, mediana, desvio padrão, coeficiente de variação e os valores mínimo e máximo (técnicas de estatística descritiva) e foram calculadas as frequências percentuais e construídas as respectivas distribuições de frequência das variantes. Foi aplicado o teste de Qui-quadrado para homogeneidade a fim de avaliar se a distribuição das variáveis diferia entre os grupos. Nos casos em que as suposições do teste Qui-quadrado não foram satisfeitas, foi aplicado o teste alternativo Exato de Fisher. Em todas as conclusões foi utilizado o nível de significância de 5\%. Os dados foram digitados na planilha do Excel e foram analisados através do programa Statistical Package for the Social Sciences (SPSS) na versão 18.

\section{RESULTADOS}

Todos os oito animais sobreviveram ao período estabelecido de 360 dias para observação clínica e realização da segunda cirurgia. Durante o período de observação, os cães não apresentaram sinais clínicos de hemorragia ou hematoma na ferida operatória. Também não foram encontrados sinais de infecção da ferida operatória ou edema nos membros operados, em ambos os grupos. Um cão apresentou dilatação das veias superficiais no membro e região inguinal esquerda (BP) (Tabela 1). A avaliação, através da dopplerfluxometria no pós-operatório, demonstrou a presença de fluxo fásico com a respiração nas veias femorais em todos os cães avaliados no primeiro dia de pós-operatório (DPO). O padrão do fluxo sofreu alteração, perdendo a característica de ser fásico com a respiração e tornando-se contínuo no grupo do BP da seguinte forma: no oitavo DPO, um cão apresentou o fluxo contínuo, no 30 DPO, dois cães e, 360 dias após a cirurgia, cinco cães. Já no grupo controle observou-se que no oitavo e 30 DPO, os cães mantiveram o fluxo fásico com a respiração, porém, 360 dias após a cirurgia, um cão apresentou fluxo de padrão contínuo (Tabela 1).

Tabela 1. Distribuição do fluxo ao Doppler, edema, dilatação venosa superficial em diferentes momentos da avaliação.

\begin{tabular}{|c|c|c|c|c|c|c|c|c|}
\hline & \multicolumn{2}{|c|}{ 1o dia } & \multicolumn{2}{|c|}{ 8o dia } & \multicolumn{2}{|c|}{30 dia } & \multicolumn{2}{|c|}{360 dia } \\
\hline & $\mathrm{BP}$ & Controle & $\mathrm{BP}$ & Controle & $\mathrm{BP}$ & Controle & $\mathrm{BP}$ & Controle \\
\hline \multicolumn{9}{|l|}{ Fluxo Fásico } \\
\hline Sim & $8(100 \%)$ & $8(100 \%)$ & $7(87,5 \%)$ & $8(100 \%)$ & $6(75 \%)$ & $8(100 \%)$ & $3(37,5 \%)$ & $7(87,5 \%)$ \\
\hline Não & $0(0 \%)$ & $0(0 \%)$ & $1(12,5 \%)$ & $0(0 \%)$ & $2(25 \%)$ & $0(0 \%)$ & $5(62,5 \%)$ & $1(12,5 \%)$ \\
\hline$p$-valor* & \multicolumn{2}{|c|}{$-* \star$} & \multicolumn{2}{|c|}{1.000} & \multicolumn{2}{|c|}{0.467} & \multicolumn{2}{|c|}{0.119} \\
\hline
\end{tabular}

\begin{tabular}{|c|c|c|c|c|c|c|c|c|}
\hline \multirow{2}{*}{\multicolumn{9}{|c|}{ Edema }} \\
\hline & & & & & & & & \\
\hline Sim & $0(0 \%)$ & $0(0 \%)$ & $0(0 \%)$ & $0(0 \%)$ & $0(0 \%)$ & $0(0 \%)$ & $0(0 \%)$ & $0(0 \%)$ \\
\hline Não & $8(100 \%)$ & $8(100 \%)$ & $8(100 \%)$ & $8(100 \%)$ & $8(100 \%)$ & $8(100 \%)$ & $8(100 \%)$ & $8(100 \%)$ \\
\hline p-valor* & \multicolumn{2}{|c|}{$-* \star$} & \multicolumn{2}{|c|}{$-* *$} & \multicolumn{2}{|c|}{$-* *$} & \multicolumn{2}{|c|}{$-* \star$} \\
\hline \multicolumn{9}{|c|}{ Dilatação Venosa Superficial } \\
\hline Sim & $0(0 \%)$ & $0(0 \%)$ & $0(0 \%)$ & $0(0 \%)$ & $0(0 \%)$ & $0(0 \%)$ & $1(12,5 \%)$ & $0(0 \%)$ \\
\hline Não & $8(100 \%)$ & $8(100 \%)$ & $8(100 \%)$ & $8(100 \%)$ & $8(100 \%)$ & $8(100 \%)$ & $7(87,5 \%)$ & $8(100 \%)$ \\
\hline$p$-valor* & \multicolumn{2}{|c|}{$-* \star$} & \multicolumn{2}{|c|}{$-* *$} & \multicolumn{2}{|c|}{$-* \star$} & \multicolumn{2}{|c|}{1.000} \\
\hline
\end{tabular}

* $p$-valor do teste exato de Fisher (se $p$-valor <0,05 a distribuição do fator em estudo é homogênea entre os momentos de análise);

** Não pode ser calculado, pois, a ausência/presença foi constante nos momentos avaliados. 
A flebografia realizada 360 dias após a primeira cirurgia demonstrou que três (37,5\%) enxertos de BP e sete $(87,5 \%)$ do grupo controle estavam pérvios (Figura 1).

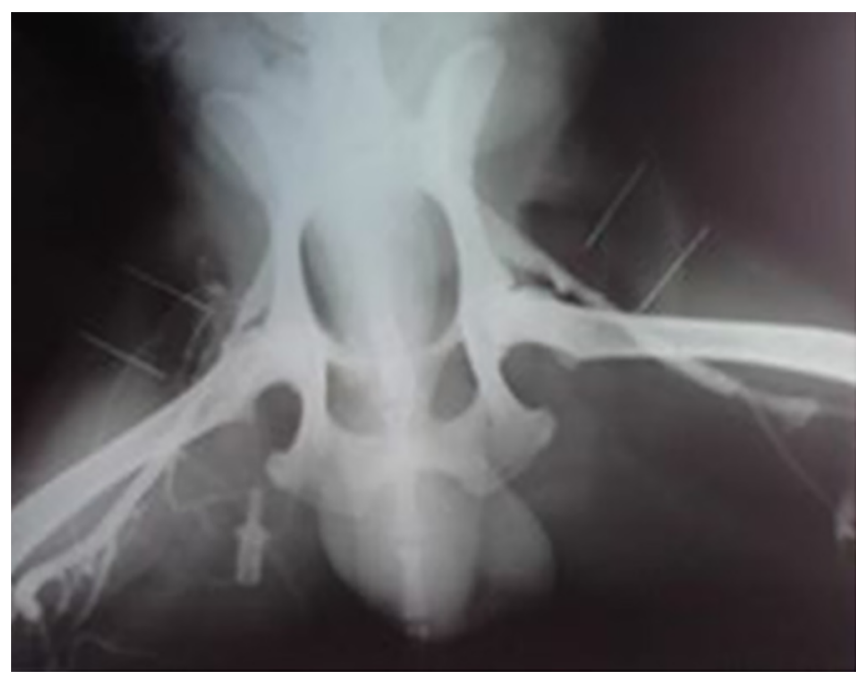

Figura 1. Flebografia após 360 dias: agulhas radiopacas destacam os enxertos.

À avaliação macroscópica, as veias femorais, nos dois grupos, estavam revestidas externamente por uma espessa camada de tecido conjuntivo frouxo e apresentavam aderência cicatricial aos tecidos vizinhos. Não foi observada modificação da coloração ou da consistência do enxerto de BP (Figura 2). A avaliação histológica dos segmentos venosos demonstrou que a camada íntima de revestimento dos enxertos, nos dois grupos, era contínua com a camada íntima da veia femoral receptora, sendo formada por uma camada espessa de tecido fibroso firmemente aderida à superfície interna dos enxertos (Figura 3). A superfície externa dos enxertos no grupo do BP era circundada por uma camada de tecido conjuntivo rica em linfócitos, neutrófilos e raros macrófagos firmemente aderida aos enxertos. Esta camada com células inflamatórias era revestida externamente por uma espessa camada de tecido fibroso. Os implantes do grupo controle estavam também revestidos externamente por uma camada de células inflamatórias, porém, estas ocorriam em menor quantidade que no grupo experimental, demonstrando uma reação inflamatória leve. No grupo controle a camada de células inflamatórias também estava revestida externamente por uma espessa camada de tecido fibroso. Não foram encontrados focos de processo degenerativo ou áreas de calcificação.

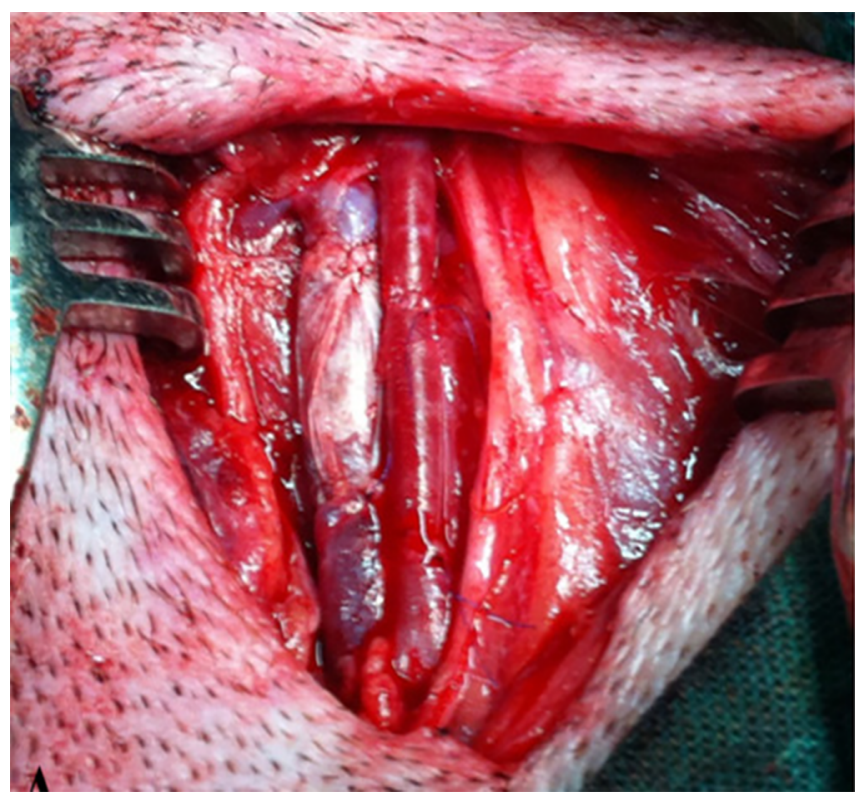

Figura 2. Aspecto da veia femoral no grupo do BP após 360 dias.

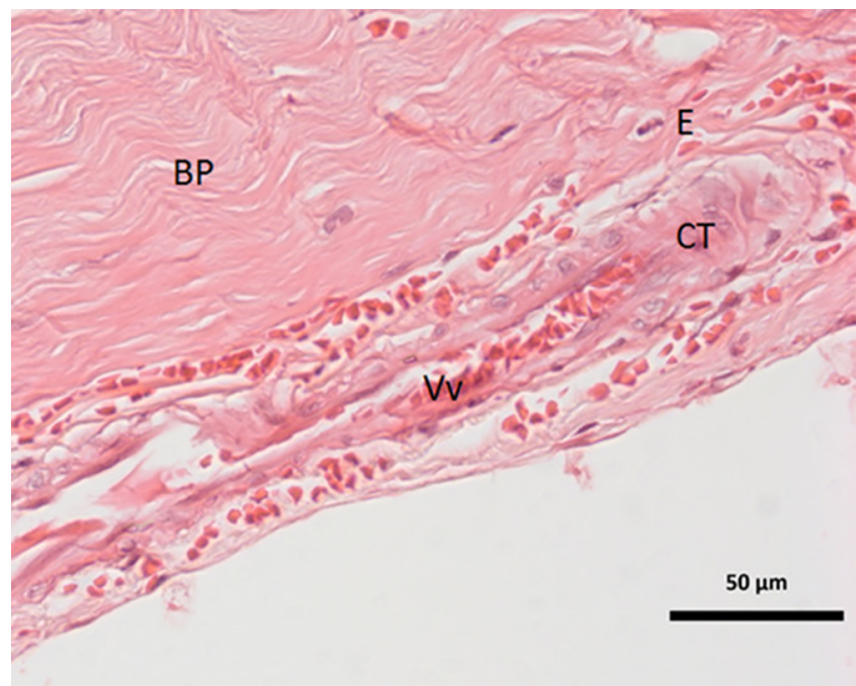

Figura 3. Superfície interna do enxerto de $B P . V_{V}$ - vasavasorum, E- endotélio, CT- tecido conectivo. 


\section{DISCUSSÃO}

Os cães têm sido utilizados como modelo experimental em diversos trabalhos científicos. Alguns autores descrevem que o comportamento das próteses vasculares nos cães é o que mais se assemelha a humanos. Isto explica a sua preferência como modelo animal habitualmente usado para avaliação de enxertos vasculares ${ }^{16-21}$.

Devido tanto à simplicidade para o acesso quanto ao seu calibre, as veias femorais foram escolhidas para a realização destes experimentos. Na exposição da veia femoral não há o trauma sobre a musculatura abdominal, que é frequentemente relacionado à dor e a limitação dos movimentos no pós-operatório, e não há manipulação dos órgãos intra-abdominais, como ocorre na cirurgia sobre a veia cava inferior. Há menor risco de alterações hemodinâmicas graves e, além disto, a ampla rede de veias colaterais nesta região garante, na maioria dos casos, a drenagem do membro quando ocorre trombose da veia femoral ${ }^{16,17,20}$.

Apesar do enxerto de BP apresentar algumas propriedades diferentes de uma veia autóloga, como por exemplo, a elasticidade menor, ainda assim, não houve dificuldade na realização da sutura. Esta característica já foi demonstrada em outros trabalhos que utilizaram a membrana do BP como patch em angioplastias de artérias e veias femorais de cães ${ }^{16,17}$.

Outro aspecto do presente estudo é que o grupo controle foi realizado na veia femoral contralateral à do implante do enxerto de BP. Isto permitiu que um menor número de animais fosse utilizado no estudo, e, consequentemente, sacrificados, o que está de acordo com os princípios bioéticos atuais. Além disto, o fato de cada animal ser seu próprio controle tornou os grupos mais homogêneos, assim como em outros trabalhos que utilizaram o mesmo princípio, como nos casos de remendos de biopolímero em veias e artérias femorais de cães, e no uso de látex como prótese em artéria femoral, também de cães ${ }^{16,17}$.

O tempo de observação dos grupos foi de 360 dias. Este período está em acordo com o observado em vários trabalhos experimentais que avaliam o uso de substitutos vasculares em modelos animais. Isto ocorre porque o processo de cicatrização dos enxertos vasculares, que envolve sua incorporação pelos tecidos orgânicos adjacentes ocorre entre 180 e 360 dias após o implante $3,20,21$.

Os exames complementares empregados neste estudo, para a avaliação dos enxertos venosos, foram a dopplerfluxometria e a flebografia ascendente. A dopplerfluxometria é um exame não invasivo que utiliza princípios da ultrassonografia para avaliar o fluxo venoso, porém não fornece informações sobre a morfologia da veia. Vários trabalhos experimentais têm demonstrado que a dopplerfluxometria pode ser utilizada nas avaliações pós-operatórias na cirurgia vascular reconstrutora. A flebografia é um exame invasivo que utiliza contraste iodado e permite estudar tanto a fisiologia quanto a morfologia venosa, por isso é considerada o padrão ouro para a avaliação do sistema venoso e tem sido utilizada amplamente em modelos experimentais que utilizam animais para testar substitutos vasculares ${ }^{16,17}$. A associação entre a avaliação clínica, a dopplerfluxometria 
e a flebografia, permitiu uma alta sensibilidade no diagnóstico da trombose e da perviedade do enxerto venoso. O uso destes exames em associação tem sido descrito em outros estudos experimentais que avaliam enxertos venosos ${ }^{16,17}$

As principais complicações decorrentes do uso dos enxertos venosos tubulares são trombose, ruptura e infecção da ferida operatória. Vários autores têm relatado, em trabalhos experimentais, a ocorrência destas complicações. A taxa de trombose encontrada na literatura referente às reconstruções que utilizaram substitutos sintéticos da veia cava varia de 20 a 30\% após um ano da cirurgia. Nas reconstruções das veias ilíacas em humanos, que apresentam menor calibre que a veia cava, utilizando próteses sintéticas de e-PTFE, é relatado uma taxa de trombose após um ano de cerca de $50 \%$ enquanto que nas reconstruções endovasculares, também das veias ilíacas, a taxa de trombose após um ano varia de 70 a $80 \%{ }^{7-10}$. Estudo de Greca et al., que utilizou patch com enxerto biológico na veia cava de cães, revelou que, após 40 dias, dos 16 cães do estudo, apenas um apresentou trombose parcial, e a anticoagulação foi realizada no período transoperatório ${ }^{3}$. No presente estudo, não foi realizada nenhum tipo de anticoagulação, o que pode ter contribuído para a maior taxa de trombose no grupo do BP.

Não foram identificados casos de infecção ou hemorragia na ferida operatória, em ambos os grupos, achados que têm sido relatados em outros trabalhos experimentais ${ }^{16,17}$. Foi observado em todos os casos do grupo controle e do grupo experimental, o desenvolvimento de reação inflamatória crônica na superfície externa e a presença de fibrose na superfície interna, de forma semelhante à encontrada na literatura ${ }^{16,17}$.
O processo de cicatrização dos enxertos de BP ocorreu de forma similar ao dos enxertos de veia autóloga no que concerne à presença de células inflamatórias na sua superfície externa e fibrose nas duas superfícies, porém no grupo experimental houve uma reação inflamatória mais importante do que no grupo controle. Não foram encontradas células fagocitárias na reação inflamatória da superfície externa, em nenhum dos enxertos de ambos os grupos. A ausência destas células inflamatórias confirma o baixo teor antigênico de ambos os enxertos, uma vez que a presença de células fagocitárias tem o objetivo de defesa do organismo diante de um agente agressor. Não foram encontrados focos de calcificação na prótese de biopolímero, como é observado nos enxertos heterólogos $20-23$.

A reação inflamatória, observada neste estudo se concentrou na periferia dos implantes, tanto no enxerto autólogo quanto na prótese de BP. Foi observado também que as camadas íntima e adventícia estavam firmemente aderidas, respectivamente, às faces interna e externa da prótese de biopolímero. Este aspecto já foi descrito em trabalhos anteriores que utilizaram remendos da membrana de BP em arterioplastias e venoplastias femoral em cães ${ }^{10,11}$.

Os avanços no tratamento cirúrgico das lesões venosas agudas crônicas vêm evoluindo lentamente. Os resultados obtidos neste estudo representam uma perspectiva para a cirurgia de reconstrução venosa em seres humanos. Por seu baixo custo, o uso dos enxertos do BP pode trazer novas possibilidades para o tratamento de diversas doenças venosas, porém, novas pesquisas devem ser realizadas para a confirmação e aperfeiçoamento dos resultados encontrados. 


\section{A B S T R A C T}

Objective: to evaluate, through Doppler flowmetry, venography, histology and clinical evolution, the use of sugarcane biopolymer (BP) tubular grafts in the reconstruction of femoral veins in dogs. Methods: we submitted eight adult dogs to femoral vein reconstruction, on the left with BP tubular graft and on the right with autologous vein. In the postoperative period, the animals underwent clinical evaluation and femoral vein Doppler flowmetry. After 360 days, we reoperated the dogs and submitted them to femoral vein phlebography with iodinated contrast. We removed the segments of the femoral veins containing the grafts and sent them for histopathological evaluation. Results: the dogs did not present hemorrhage, hematoma, surgical wound infection or operated limb edema. One animal had superficial venous dilatation in the left inguinal region. Phlebography performed 360 days after the first surgery showed that three (37.5\%) BP grafts and seven (87.5\%) grafts from the control group (C) were patent. In the histopathological evaluation, we found an inflammatory reaction, with neutrophils and lymphocytes on the external surface of both groups. In the intimal layer of the grafts and in the outer layer in the two groups, we observed fibrosis. Conclusion: based on the results obtained with the experimental model used, BP presents potential to be used as a tubular graft for venous revascularization. However, new research must be performed to confirm its efficacy in the revascularization of medium and large diameter veins, which could allow its use in clinical practice.

Keywords: Vascular Grafting. Femoral Vein. Saccharum. Bioprosthesis. Vascular Surgical Procedures. Dogs.

\section{REFERÊNCIAS}

1. Lantz GC, Badylak SF, Coffey AC, Geddes LA, Sandusky GE. Small intestinal submucosa as a superior vena cava graft in the dog. J Surg Res. 1992;53(2):175-81.

2. D'Andrilli A, Ciccone AM, Ibrahim M, Venuta $F$, Rendina EA. A new technique for prosthetic reconstruction of the superior vena cava. J Thorac Cardiovasc Surg. 2006;132(1):192-4.

3. Greca FH, Noronha L, Costa FDA, Souza Filho ZA, Soccol AT, Feres NA, et al. Estudo comparativo da biocompatibilidade da submucosa intestinal porcina e pericárdio bovino como enxertos na veia cava de cães. Acta Cir Bras. 2005;20(4):317-22.

4. Eberhardt RT, Raffetto JD. Chronic venous insufficiency. Circulation. 2014;130(4):333-46.

5. Daenens K, Fourneau I, Nevelsteen A. Ten-year experience in autogenous reconstruction with the femoral vein in the treatment of aortofemoral prosthetic infection. Eur J Vasc Endovasc Surg. 2003;25(3):240-5.

6. Florek HJ, Brunkwall J, Orend KH, Handley I, Pribble J, Dieck R. Results from a First-in-Human trial of a novel vascular sealant. Front Surg. 2015;2:29.

7. Caroll S, Moll S. Inferior vena cava filters, MayThurner syndrome, and veins stents. Circulation. 2016;133(6):e383-7.

8. Erben $Y$, Bjarnason $H$, Oladottir GL, McBane RD, Gloviczki P. Endovascular recanalization for nonmalignant obstruction of the inferior vena cava. J Vasc Surg Venous Lymphat Disord. 2018;6(2):173-82.

9. Sfyroeras GS, Antonopoulos CN, Mantas G, Moulakakis KG, Kakisis JD, Brountzos E, et al. A review of open and endovascular treatment of superior vena cava syndrome of benign aetiology. Eur J Vasc Endovas Surg. 2017;53(2):238-54.

10. Campbell CD, Goldfarb D, Roe R. A small arterial substitute: expanded microporous polytetrafluoroethylene: patency versus porosity. Ann Surg. 1975;182(2):138-43.

11. Xue L, Greisler HP. Biomaterials in the development and future of vascular grafts. J Vasc Surg. 2003;37(2):472-80.

12. Daghighi S, Sjollema J, van der Mei HC, Busscher $\mathrm{HJ}$, Rochford ET. Infection resistance of degradable versus non-degradable biomaterials: an assessment of the potential mechanisms. Biomaterials. 2013;34(33):8013-7.

13. Fernandes Teixeira FM, Figueiredo Pereira $M$, Gomes Ferreira NL, Miranda GM, Andrade Aguiar JL. Spongy film of cellulosic polysaccharide as a dressing for aphthous stomatitis treatment in rabbits. Acta Cir Bras. 2014;29(4):231-6.

14. Paterson-Beedle M, Kennedy JF, Melo FAD, Lloyd LL, Medeiros V. A cellulosic exopolysaccharide produced from sugarcane molasses by a Zoogloea sp. Carbohydr Polym. 2000;42(4):375-83.

15. Martins AG, Lima SV, Araújo LA, Vilar FO, Cavalcante NT. A wet dressing for hypospadias surgery. Int Braz J Urol. 2013;39(3):408-13. 
16. Aguiar JL, Lins EM, Marques SR, Coelho AR, Rossiter RO, Melo RJ. Surgarcane biopolymer patch in femoral artery angioplasty on dogs. Acta Cir Bras. 2007;22 Suppl 1:77-81.

17. de Barros-Marques SR, Marques-Lins E, de Albuquerque MC, de Andrade-Aguiar JL. Sugarcane biopolymer patch in femoral vein angioplasty on dogs. J Vasc Surg. 2012;55(2):517-21.

18. Berenstein A, Song JK, Tsumoto T, Namba K, Niimi $Y$. Treatment of experimental aneurysms with an embolic-containing device and liquid embolic agent: feasibility and angiographic and histological results. Neurosurgery. 2009;64(2):367-73.

19. Divakov MG. Revascularization of avascular spongy bone and head of the femur in transplantation of vascular bundle (An experimental and clinical study). Acta Chir Plast. 1991;33(2):114-25.

20. Teijeira FJ, Marois $Y$, Aguiar L, Guidoin R, Bauset $\mathrm{R}$, Lamoureux $\mathrm{G}$, et al. Comparison of processed bovine internal mammary arteries and autologous veins as arterial femoral substitutes in dogs: blood compatibility and pathological characteristics. Can J Surg. 1989;32(3):180-7.

21. Benzel EC, McMillan R, Fowler MR, Landreneau MD, Kesterson L, Payne DL. Histological comparison of autogenous canine fascia lata, Gore-Tex, lyophilized human fascia lata, and autogenous canine vein for vascular patch graft material in a canine arteriotomy model. Neurosurgery. 1992;31(1):108-13.

22. Douglas JF, Gaughran ER, Henderson J, Lord GH, Rosenberg N. The use of arterial implants prepared by enzymatic modification of arterial heterografts: II. The physical properties of the elastica and collagen components of the arterial wall. AMA Arch Surg. 1957;74(1):89-95.

23. Robotin-Johnson MC, Swanson PE, Johnson DC, Schuessler RB, Cox JL. An experimental model of small intestinal submucosa as a growing vascular graft. J Thorac Cardiovasc Surg. 1998;116(5):805-11.

Recebido em: 04/01/2018

Aceito para publicação em: 17/05/2018

Conflito de interesse: nenhum.

Fonte de financiamento: nenhuma.

\section{Endereço para correspondência:}

Esdras Marques Lins

E-mail: esdraslins@uol.com.br esdraslins39@gmail.com

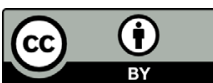

